\title{
IMPACTO DE LA PANDEMIA POR SARS-COV-2 EN ADOLESCENTES QUE VIVEN CON EL VIH EN LIMA, PERÚ
}

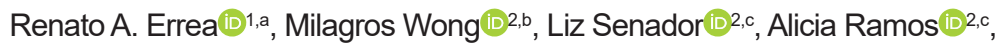

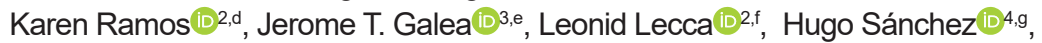 \\ Carlos Benites (iD,h, Molly F. Franke (D)1,i \\ 1 Departamento de Salud Global y Medicina Social, Escuela de Medicina de Harvard, Boston, Estados Unidos de América. \\ 2 Socios en Salud, Lima, Perú. \\ 3 Escuela de Trabajo Social, Universidad del Sur de Florida, Tampa, Estados Unidos de América. \\ ${ }^{4}$ Epicentro Salud, Lima, Perú. \\ ${ }^{5}$ Dirección de Prevención y Control del VIH, ITS y Hepatitis, Ministerio de Salud del Perú, Lima, Perú. \\ a Médico cirujano, maestro en Ciencias Médicas de Salud Global; ${ }^{b}$ licenciada en Enfermería; ${ }^{c}$ técnica en Enfermería; \\ d licenciada en Nutrición Humana, maestra en Gerencia de Proyectos y Programas Sociales; ${ }^{\mathrm{e}}$ bachiller en Psicología, doctor \\ en Ciencias de Prevención de VIH; ${ }^{\mathrm{f}}$ médico cirujano, maestro en Epidemiologia; ${ }^{\mathrm{g}}$ licenciado en Psicología; ${ }^{\mathrm{h}}$ médico \\ especialista en Infectología, maestro en Salud Pública; i bachiller en Sociología, doctora en Epidemiología.
}

\section{RESUMEN}

Los adolescentes que viven con el VIH (AVVIH) tienen tasas más bajas de supresión virológica y tasas más altas de deterioro inmunológico en comparación con sus contrapartes mayores, colocándolos potencialmente en alto riesgo de enfermedad severa por SARS-CoV-2. Los AVVIH que están transicionando hacia el cuidado de adultos enfrentan dificultades adicionales para permanecer en el cuidado y adherirse al tratamiento antirretroviral. En esta sección especial reportamos el impacto observado en AVVIH transicionando al servicio de VIH para adultos durante la pandemia por SARS-CoV-2. En primer lugar, el acceso a la atención del VIH se ha visto perjudicado por la restricción del transporte público, la falta de antirretrovirales, y la suspensión de citas médicas y laboratoriales. Adicionalmente, dificultades financieras, incertidumbre sobre el futuro, estresores emocionales, alteración del estilo de vida y el temor de divulgar el diagnóstico involuntariamente han desafiado aún más la continuidad en la atención de esta población adolescente.

Palabras clave: VIH; Adolescente; SARS-CoV-2; COVID-19; Impactos en la Salud; Accesibilidad a los Servicios de Salud; Salud Mental (fuente: DeCS BIREME).

\section{IMPACT OF SARS-COV-2 PANDEMIC ON ADOLESCENTS LIVING WITH HIV IN LIMA, PERU}

\begin{abstract}
Adolescents living with HIV (ALWH) have lower rates of virologic suppression and higher rates of immunologic decline compared to their older counterparts, potentially placing them at high-risk for developing severe SARS-CoV-2 disease. ALWH who are transitioning to adult care face additional challenges to remaining in care and adhering to treatment. In this special section we report the experiences of ALWH in the process of transitioning to adult HIV services during the COVID-19 pandemic. In first place, the government-mandated stay-at-home order has substantially limited access to full HIV care by restricting public transportation, HIV medication stock-outs, and the suspension of routine medical and laboratory appointments. In addition, financial hardship, uncertainty about their future plans, emotional stressors, lifestyle disruptions, and concerns of involuntary disclosure have further challenged continuity in care for this adolescent population.
\end{abstract}

Keywords: HIV; Adolescent; SARS-CoV-2; COVID-19; Health Impact; Healthcare Access; Mental Health (source: MeSH NLM).

\section{INTRODUCCIÓN}

Las personas que viven con VIH no controlado pueden tener un mayor riesgo de enfermedad severa causada por el SARS-CoV-2 (COVID-19) ${ }^{(1)}$. Los adolescentes que viven con el VIH (AVVIH) tienen tasas más bajas de supresión virológica ${ }^{(2,3)}$ y tasas más altas de deterioro inmunológico en comparación con poblaciones de mayor edad ${ }^{(3)}$, debido en parte a una menor adherencia al 
tratamiento antirretroviral (TARV) ${ }^{(2,4,5)}$. Además, para los AVVIH que están ingresando a la atención de adultos - ya sea derivados del servicio pediátrico o debido a que cumplieron con la edad límite para seguir atendiéndose en el servicio de pediatría-, el nuevo entorno, muchas veces poco amigable de la atención médica de adultos, el desconocimiento de los procedimientos administrativos para comenzar a atenderse en el servicio de adultos, y las propias características de la adolescencia; aumentan aún más el riesgo de no adherirse al TARV y de interrumpir la atención ${ }^{(6,7)}$. Por ello, entre las personas que viven con el $\mathrm{VIH}$, los adolescentes pueden encontrarse en una especial situación de vulnerabilidad durante la pandemia de la COVID-19.

En el 2019, iniciamos un estudio piloto para evaluar la viabilidad de una intervención para apoyar a los AVVIH a medida que realizan la transición hacia el servicio de VIH de adultos en hospitales públicos en Lima, Perú. La intervención consistió en acompañamiento a las citas médicas, sesiones de apoyo entre pares, tratamiento directamente observado (DOT) para aquellos con adherencia subóptima, y navegación del sistema de salud para facilitar el cumplimiento de los requisitos administrativos (ej., trámites para afiliarse al seguro de salud público). La intervención incluye seis meses de apoyo intensivo, seguido de una fase de tres meses de reducción progresiva del apoyo brindado. Como parte del estudio, un comitéasesor juvenil (CAJ) formado también por AVVIH, ofrece recomendaciones para mejorar las intervenciones del estudio. En enero del 2020, se completó el enrolamiento de 30 AVVIH (entre 15 a 21 años) con diversas características médicas - antecedente de abandono a TARV, en tratamiento para tuberculosis y en situación de embarazo- y sociales - minorías sexuales o de género, población inmigrante venezolana, viviendo en condición de pobreza, y carencia de apoyo de los padres- (Tabla 1).

Tras la pandemia por SARS-CoV-2, el gobierno peruano estableció rápidamente estrictas medidas preventivas para controlar la propagación del virus, las cuales incluyeron permanencia obligatoria en el hogar, toques de queda y cierre de fronteras ${ }^{(8)}$. A pesar de ello, Perú es un epicentro de la pandemia en América del Sur y en todo el mundo, con más de un millón de casos confirmados y el mayor número de fallecidos por cien mil habitantes en todo el mundo ${ }^{(9)}$.

En este artículo, reportamos nuestra experiencia sobre el impacto de la pandemia en los 30 AVVIH de nuestro estudio y los adolescentes participantes del CAJ. La información proporcionada en este artículo surge de la interacción de distintos miembros del equipo de investigación -principalmente, la coordinadora del estudio, las supervisoras de DOT y el facilitador de sesiones entre pares- con los adolescentes, tanto durante las actividades formales del estudio de investigación (acompañamiento a recojo de medicamentos, llamadas de seguimiento de DOT y sesiones con el CAJ) como a través de las conversaciones informales (conversaciones telefónicas o de WhatsApp de coordinación de actividades) que
Tabla 1. Características de 30 adolescentes que viven con VIH incluidos en el estudio.

\begin{tabular}{lc}
\hline Característica & $\mathbf{n}(\%)$ \\
\hline Edad (rango) & $15-21$ años \\
Sexo femenino & $12(40,0)$ \\
Condiciones socio-médicas de importancia & \\
$\quad \begin{array}{l}\text { Historia de abandono de tratamiento antirret- } \\
\text { roviral }\end{array}$ & $12(40,0)$ \\
$\quad$ Tuberculosis en tratamiento & $2(6,7)$ \\
$\quad$ Embarazo & $4(13,3)$ \\
Otras condiciones de vulnerabilidad social & \\
Viviendo en condición de pobreza & $22(73,3)$ \\
Perteneciente a minorías sexuales o de género & $11(36,7)$ \\
Inmigrante venezolano & $5(16,7)$ \\
Orfandad & $12(40,0)$ \\
\hline
\end{tabular}

surgen naturalmente tras la comunicación constante y cercana entre el equipo investigador de campo y los participantes. Dichas interacciones son importantes para captar y entender las diversas necesidades de los participantes. Los tópicos reportados a continuación representan los temas que más sobresalieron durante aquellas actividades y conversaciones, debido a su recurrencia y grado de afectación en los adolescentes. Además, comentamos como adaptamos las actividades del estudio para cubrir las necesidades de los AVVIH.

El estudio piloto del que deriva el presente artículo fue aprobado para su realización por los Comités de Ética del Instituto Nacional de Salud del Niño, del Hospital Nacional Arzobispo Loayza, del Hospital Nacional Hipólito Unanue, y de la Universidad de Harvard. Los adolescentes de dicho estudio brindaron su consentimiento informado para participar en el mismo.

\section{IMPACTO EN LOS ADOLESCENTES QUE VIVEN CON EL VIH}

\section{Acceso limitado a los servicios de salud}

La mayoría de los pacientes que toman TARV en el Perú obtienen los medicamentos en hospitales públicos del tercer nivel de atención mayoritariamente centralizados en las áreas más urbanas. Las medidas de salud pública contra el SARSCoV-2 incluyeron acceso restringido a los hospitales, tanto para minimizar la propagación nosocomial del SARS-CoV-2 como para permitir a los proveedores de salud priorizar la atención de pacientes con la COVID-19. De esa manera, la atención del VIH se limitó al reabastecimiento del TARV, mientras que las citas médicas, los controles de laboratorio y las actividades administrativas relacionadas fueron canceladas. 
Estas restricciones significaron múltiples dificultades para los AVVIH en proceso de transición a la atención de adultos. Por ejemplo, a un AVVIH que recientemente había sido transferido al servicio de adulto, se le negó la entrega de TARV en el hospital debido a que los servicios administrativos para incluir a un nuevo paciente al Programa de VIH del hospital no se encontraban disponibles durante la pandemia. El participante pudo recibir su TARV en el hospital solo después de que un facilitador de nuestro estudio y el médico pediatra del hospital de origen intercedieran por él.

Otro AVVIH, que días antes de la cuarentena obligatoria había reiniciado tratamiento después de dos años sin TARV y un largo historial de adherencia subóptima, no pudo realizarse el control de su carga viral programada para estas fechas. El paciente, consciente de su historial de mala adherencia, expresó mucha preocupación por la posible resistencia al TARV y el no poder confirmar la efectividad de su régimen antirretroviral actual. Adicionalmente, la cancelación de las consultas externas no fue específica para la atención del VIH; los AVVIH se vieron también afectados por no poder recibir atención de otras infecciones de trasmisión sexual concomitantes, atención de salud mental y/o atención posparto.

\section{Escasez de TARV}

Algunos adolescentes del estudio reportaron escasez de los medicamentos antirretrovirales en los hospitales donde reciben tratamiento. Tras agotarse los medicamentos, una adolescente informó que para que no se interrumpiese su tratamiento, un consejero del Programa de VIH del establecimiento donde ella acude, le tuvo que prestar de sus propias medicinas. Otros adolescentes informaron que sus regímenes sí llegaron a ser cambiados debido a la escasez de medicinas. Estos cambios de régimen generaron preocupación en dichos adolescentes por temor a la aparición de eventos adversos, los cuales son más comunes al iniciar un nuevo régimen antirretroviral y que pueden ser difíciles de identificar o manejar en ausencia de consultas médicas.

\section{Dificultad para transportarse}

Ir a los hospitales durante la pandemia para recoger el TARV significó un gran desafío para los AVVIH. En primer lugar, el restringido horario y unidades de transporte público redujeron las posibilidades de acudir a los hospitales. Por ejemplo, al prohibir la circulación de medios de transporte como «combis» $\mathrm{y}$ «mototaxis», los adolescentes de nuestro estudio, que en su mayoría viven en las afueras o áreas de difícil acceso a la ciudad, se vieron directamente afectados ya que dependen de estos servicios de transporte para llegar a los paraderos de buses más cercanos. De esa manera, algunos AVVIH informaron tener que caminar varios kilómetros para llegar al paradero y esperar largas horas para tomar el próximo bus.

Un segundo desafío fue mantenerse protegido de la exposición al SARS-CoV-2 dentro del transporte público, el cual ya ha sido previamente reportado como un factor de riesgo para el contagio de otras enfermedades infecciosas respiratorias en Perú ${ }^{(10,11)}$. Así, los adolescentes informaron sentirse inseguros en el transporte público debido al hacinamiento y al uso inconsistente de mascarillas o protectores faciales entre los usuarios. Además, señalaron temer que su propia exposición podría también poner en riesgo a los demás familiares con los que viven. Esto último fue de especial preocupación en AVVIH infectados perinatalmente y ahora huérfanos, cuyos cuidadores suelen ser los abuelos $\mathrm{u}$ otras parientes de edad avanzada.

\section{Dificultades financieras e incertidumbre sobre el futuro}

Los AVVIH también han sufrido las consecuencias económicas de las medidas preventivas tomadas por la COVID-19 ${ }^{(12)}$, ya sea por la pérdida de sus propios trabajos o el de sus cuidadores. Adolescentes que dependían de trabajos informales, como vendedores ambulantes o venta en carros de comida, se vieron totalmente impedidos de laborar tras las órdenes de distanciamiento social obligatorio. De ese modo, un adolescente de nuestro estudio se vio forzado a mudarse a una vivienda más económica por no poder seguir pagando el alquiler de la vivienda donde residía. Otros adolescentes que dependían económicamente de cuidadores que perdieron el empleo, vieron interrumpidos sus planes de iniciar o continuar estudiando. Esto es especialmente importante en AVVIH, ya que estudios previos han reportado que el tener planes sobre el futuro promueve la adherencia al TARV ${ }^{(13)}$. Por ello, la incertidumbre sobre su educación y futuro puede impactar negativamente la salud mental de los AVVIH y su estado de salud relacionado al VIH.

\section{Depresión, aislamiento y alteración del estilo de vida}

$\mathrm{El}$ aislamiento por periodos prolongados ha sido asociado en otros estudios con un aumento de los trastornos de salud mental (particularmente depresión) ${ }^{(14)}$, y con la alteración del estilo de vida ${ }^{(15)}$; ambas situaciones, a su vez, impactan negativamente en la salud de las personas que viven con $\mathrm{VIH}^{(16,17)}$. En nuestro estudio, los AVVIH expresaron tener constantes sentimientos de tristeza en parte debido a la disminución de las interacciones con amigos, incluyendo las nuevas amistades que formaron con otros participantes del estudio. Otros informaron que las ya complicadas relaciones familiares que tenían se agravaron al pasar más tiempo en casa. Por ejemplo, un adolescente que vivía con parientes reportó sufrir mayor agresión verbal y hasta miedo de ser agredido físicamente por sus familiares. Por otra parte, algunos AVVIH experimentaron un aumento del sueño que alteró su rutina diaria e interrumpió la toma constante de los antirretrovirales y el DOT.

Miedo a revelar el diagnóstico involuntariamente Las medidas de salud pública también han ocasionado mayor riesgo de divulgar el estado de VIH del adolescente. Por 
ejemplo, un miembro del CAJ describió su experiencia en el establecimiento de salud donde se atendía por el VIH; para minimizar la transmisión del SARS-CoV-2, el establecimiento restringió el aforo dentro del local, lo que condujo a largas colas fuera del establecimiento. Ello conllevó a que algunos pacientes se negaran a hacer cola en la calle por temor a ser vistos, regresando a sus hogares sin haberse reabastecido de sus antirretrovirales. Asimismo, para los AVVIH que vivían con familiares que desconocían el estado de VIH, la necesidad de ir al hospital para recoger el TARV ha causado que aquellos que desean mantener confidencial su diagnóstico ideen justificaciones alternas para sustentar quebrar las normas de aislamiento impuestas por el gobierno.

\section{APOYO PROPORCIONADO A LOS ADOLESCENTES QUE VIVEN CON EL VIH}

Tras la pandemia, las actividades de intervención del estudio tuvieron que ser adaptadas para seguir brindando apoyo a los AVVIH. Para ello fue necesario tener en cuenta las distintas necesidades de cada participante y las diversas circunstancias en las que cada uno de ellos vive. Para empezar, implementamos llamadas telefónicas semanales con los AVVIH para coordinar las próximas fechas de recojo del TARV, preparar un plan acerca de cómo llegar al establecimiento de salud durante las restricciones de movilización y transporte, y para conversar sobre cualquier desafío nuevo que estén presentando debido a la pandemia.

En segundo lugar, el DOT, que originalmente se hacía en persona en la casa del participante, se empezó a realizar a través de videollamada para garantizar el cumplimiento diario del tratamiento en los AVVIH con antecedentes de no adherencia. Algunos AVVIH optaron por realizar video llamadas de DOT en grupos pequeños para aprovechar en interactuar con otros adolescentes participantes y con el personal del estudio.

Tercero, hicimos uso de las redes sociales para reforzar la interacción y educación entre los participantes. Tres veces a la semana se realizan sesiones privadas de Facebook Live. En cada sesión, un presentador invitado toca un tema en particular, por ejemplo, sexo seguro, relaciones de pareja saludables, amor propio/autoestima, y prevención de la infección por SARS-CoV-2. En dichas sesiones, los adolescentes pueden interactuar con el ponente y con el resto de los participantes en tiempo real a través del chat, o pueden ver la grabación de la sesión en cualquier otro momento si es que no pudieron estar presente en la transmisión en vivo.

Adicionalmente, a través de un grupo de WhatsApp y concursos de Tik Tok, los participantes mantienen comunicación activa entre ellos y con el equipo del estudio. A los adolescentes con acceso limitado a Internet, se les otorga crédito telefónico semanalmente, para que puedan participar en las diferentes actividades virtuales. Al respecto de las actividades, los adolescentes han comentado que éstas les ayudan a mantenerse enfocados en su bienestar y motivados a pesar de la difícil circunstancia de la pandemia. No obstante, si bien la participación en las actividades virtuales y en línea ha sido alta, el hacinamiento y la falta de privacidad en el hogar han llevado a algunos adolescentes, especialmente a aquellos preocupados por la divulgación involuntaria del estado de VIH en el hogar, a optar por no participar en las actividades.

Finalmente, un grupo de AVVIH con urgentes necesidades médicas o de salud mental, ha podido recibir atención a través de telemedicina o consultas telefónicas con profesionales médicos del sector privado que ofrecieron voluntariamente su tiempo. Dichas sesiones fueron coordinadas y facilitadas por el personal del estudio.

\section{CONCLUSIONES}

Las barreras estructurales (problemas de transporte y escasez de medicamentos) ${ }^{(18-20)}$, la pobreza ${ }^{(21)}$, el estigma ${ }^{(22)}$, y las enfermedades mentales ${ }^{(16)}$ impiden el acceso y la continuidad de la atención en salud en diversos países, incluido el Perú ${ }^{(23-25)}$. El SARS-CoV-2 y las importantes respuestas de salud pública han intensificado estas barreras, comprometiendo directamente los múltiples componentes del acceso a los servicios de salud como la entrada a los establecimientos y la oferta oportuna y de calidad de los servicios adecuados con el personal capacitado ${ }^{(26)}$. Asimismo, los AVVIH, especialmente aquellos en transición a la atención del VIH en adultos, pueden ser más vulnerables a la COVID-19 y a las consecuencias indirectas socioeconómicas y sobre la salud mental generadas por las medidas preventivas tomadas por el gobierno. A medida que esta pandemia continúa en Perú y en otras partes de América Latina y el mundo, se necesitan estrategias de salud pública para garantizar la salud y el bienestar a largo plazo de los adolescentes.

Posibles soluciones para garantizar la atención a las personas que viven con VIH durante las actuales circunstancias incluyen el uso de telemedicina (iniciativa que el Ministerio de Salud del Perú ha comenzado a implementar) y la oferta de servicios diferenciados del VIH, que incluyen, por ejemplo, la distribución de TARV en establecimientos de salud del primer nivel de atención y más cercanos a los hogares de los pacientes, o el envío de los medicamentos a los domicilios ${ }^{(27-29)}$. Además, el fortalecimiento del capital social, entendido como los recursos de apoyo proveídos por las propias redes de contacto, cobra especial importancia para superar las diversas dificultades de acceso a la salud a consecuencia de la pandemia; se ha descrito previamente al capital social en población joven viviendo con VIH como un potencial factor para mejorar la continuidad en la atención del $\mathrm{VIH}^{\left({ }^{(30)}\right.}$. Por último, particularmente en adolescentes, el uso de herramientas amigables como las redes 
sociales, pueden contribuir a lograr una adherencia adecuada al TARV y asegurar el bienestar en los AVVIH, durante la pandemia e incluso luego de ella.

Contribuciones de los autores: RAE y MFF redactaron el primer borrador del artículo. MW, LS, AR, HS y KR proporcionaron información clave sobre la experiencia en el campo. JTG, LL y CB aportaron significativamente al artículo desde su experticia en el campo de salud pública, epidemiología y VIH. Todos los autores

\section{REFERENCIAS BIBLIOGRÁFICAS}

1. Infectious Diseases Society of America. COVID-19: Special Considerations for People Living with HIV [Internet] Arlington: Infectious Diseases Society of America; 2020 [citado el 18 de mayo de 2020]. Disponible en: https://www.idsociety.org/globalassets/covid-19-special-considerations.

2. Kapogiannis BG, Koenig LJ, Xu J, Mayer KH, Loeb J, Greenberg L, et al. The HIV continuum of care for adolescents and young adults attending 13 urban US HIV care centers of the NICHD-ATN-CDC-HRSA SMILE Collaborative. Acquir Immune Defic Syndr. 2020;84:92-100. doi: 10.1097/ QAI.0000000000002308.

3. Nachega JB, Hislop M, Nguyen H, Dowdy DW, Chaisson RE, Regensberg $\mathrm{L}$, et al. Antiretroviral therapy adherence, virologic and immunologic outcomes in adolescents compared with adults in southern Africa. J Acquir Immune Defic Syndr. 2009;51(1):65-71. doi: 10.1097/ QAI.0b013e318199072e.

4. Rodriguez CA, Kolevic L, Ramos A, Wong M, Muñoz M, Patel K, et al. Lifetime changes in CD4 T-cell count, viral load suppression and adherence among adolescents living with HIV in urban Peru. Pediatr Infect Dis J. 2020;39:54-6. doi: 10.1097/INF.0000000000002501.

5. Auld AF, Agolory SG, Shiraishi RW, Wabwire-Mangen F, Kwesigabo G, Mulenga $\mathrm{M}$, et al. Antiretroviral therapy enrollment characteristics and outcomes among HIV-infected adolescents and young adults compared with older adults--seven African countries, 2004-2013. MMWR Morb Mortal Wkly Rep. 2014;63(47):1097-103.

6. Agwu AL, Lee L, Fleishman JA, Voss C, Yehia BR, Althoff KN, et al. Aging and loss to follow-up among youth living with human immunodeficiency virus in the HIV Research Network. J Adolesc Health. 2015;56(3):345-51. doi: 10.1016/j.jadohealth.2014.11.009.

7. Zanoni BC, Mayer KH. The adolescent and young adult HIV cascade of care in the United States: exaggerated health disparities. AIDS Patient Care STDS. 2014;28(3):128-35. doi: 10.1089/apc.2013.0345.

8. Decreto Supremo que declara Estado de Emergencia Nacional por las graves circunstancias que afectan la vida de la Nación a consecuencia del brote del COVID-19; [Internet]. Diario el Peruano. 15 marzo 2020 [citado 9 de mayo del 2020] Disponible en: https://busquedas.elperuano. pe/download/url/decreto-supremo-que-declara-estado-de-emergencia-nacional-po-decreto-supremo-n-044-2020-pcm-1864948-2.

9. Universidad de Johns Hopkins [Internet]. Baltimore: JHU; 2020. [citado el 25 de enero del 2021]. Disponible en: https://coronavirus.jhu.edu/ data/mortality.

10. Horna-Campos OJ, Consiglio E, Sánchez-Pérez HJ, Navarro A, Caylà JA, Martín-Mateo M. Pulmonary tuberculosis infection among workers in the informal public transport sector in Lima, Peru. Occup Environ Med. 2011;68(2):163-5. doi: 10.1136/oem.2009.051128.

11. Zamudio C, Krapp F, Choi HW, Shah L, Ciampi A, Gotuzzo E, et al. Public transportation and tuberculosis transmission in a high incidence setting. PLoS One. 2015;10(2):e0115230. doi: 10.1371/journal.pone.0115230.

12. Organización de las Naciones Unidas. Geneva: United Nations Labour Agency; 2020. [citado el 20 de mayo de 2020] Disponible en: https://news. un.org/en/story/2020/04/1062792. revisaron críticamente el manuscrito y aprobaron el borrador final. MFP obtuvo financiamiento para el estudio.

Financiamiento: Este estudio fue financiado por el Instituto Nacional de Alergias y Enfermedades Infecciosas, de los Institutos Nacionales de Salud de los Estados Unidos de América. Número de subvención: R21AI143365.

Conflictos de interés: Todos los autores declaran no tener ningún conflicto de interés.
13. Galea JT, Wong M, Muñoz M, Valle E, Leon SR, Díaz-Perez D, et al. Barriers and facilitators to antiretroviral therapy adherence among Peruvian adolescents living with HIV: a qualitative study. PLoS ONE. 2018;13(2):e0192791. doi: 10.1371/journal.pone.0192791.

14. Röhr S, Müller F, Jung F, Apfelbacher C, Seidler A, Riedel-Heller SG. Psychosocial impact of quarantine measures during serious coronavirus outbreaks: a rapid review. Psychiatr Prax. 2020;47(4):179-89. doi: 10.1371/ journal.pone.0192791.

15. Wang G, Zhang Y, Zhao J, Zhang J, Jiang F. Mitigate the effects of home confinement on children during the COVID-19 outbreak. Lancet. 2020;395:945-7. doi: 10.1016/S0140-6736(20)30547-X.

16. Magidson JF, Blashill AJ, Safren SA, Wagner GJ. Depressive symptoms, lifestyle structure, and ART adherence among HIV-infected individuals: a longitudinal mediation analysis. AIDS Behav. 2015;19(1):34-40. doi: 10.1007/s10461-014-0802-3

17. Rooks-PeckCR, Adegbite AH, Wichser ME, Ramshaw R, Mullins MM, Higa $\mathrm{D}$, et al. Mental health and retention in HIV care: a systematic review and meta-analysis. Health Psychol. 2018;37(6):574-85. doi: 10.1037/hea0000606.

18. Organización Mundial de la Salud. Medicines Shortages: Global approaches to addressing shortages of essential medicines in health systems. WHO Drug Information. 2016;30(2):180-5.

19. Syed ST, Gerber BS, Sharp LK. Traveling towards disease: transportation barriers to health care access. J Community Health. 2013;38(5):976-93. doi: 10.1007/s10900-013-9681-1.

20. Taber JM, Leyva B, Persoskie A. Why do people avoid medical care? A qualitative study using national data. J Gen Intern Med. 2014;30(3):290-7. doi: 10.1007/s11606-014-3089-1.

21. Organización Mundial de la Salud. Poverty Reduction Strategy Papers: Their significance for health: second synthesis report [Internet] Ginebra: OMS; 2004 [citado el 06 de junio del 2020]. Disponible en: https://www. who.int/hdp/en/prsp.pdf.

22. Pantelic M, Casale M, Cluver L, Toska E, Moshabela M. Multiple forms of discrimination and internalized stigma compromise retention in HIV care among adolescents: findings from a South African cohort. J Int AIDS Soc. 2020;23:e25488. doi: 10.1002/jia2.25488.

23. Bayer AM, Díaz CM, Chiappe M, Baker AN, Egoavil MS, Pérez-Lu JE, et al. The odyssey of linking to and staying in HIV care among male sex workers in Peru. J HIV AIDS. 2017;3(1):10.16966/2380-5536.134. doi: 10.16966/2380-5536.134.

24. Moore N, Blouin B, Razuri H, Casapia M, Gyorkos TW. Determinants of first trimester attendance at antenatal care clinics in the Amazon region of Peru: a case-control study. PLoS ONE. 2017;12(2):e0171136. doi: 10.1371/ journal.pone. 0171136 .

25. Coit J, Wong M, Galea JT, Mendoza M, Marin H, Tovar M, et al. Uncovering reasons behind treatment initiation delays among children with TB in Lima, Peru. 2020. Int J Tuberc Lung D. In Press.

26. Department of Health and Human Services, Office of Disease Prevention and Health Promotion [internet] Washington, DC: ODPHD; 2016 [citado 
el 23 de setiembre de 2020]. Disponible en: http://www.healthypeople. gov/2020/topics-objectives/topic/Access-to-Health-Services.

27. Ballester-Arnal R, Gil-Llario MD. The virus that changed Spain: impact of COVID-19 on people with HIV. AIDS Behav. 2020;1-5. doi: 10.1007/s10461-020-02877-3.

28. Ridgway JP, Schmitt J, Friedman E, Taylor M, Devlin S, McNulty M, et al. HIV care continuum and COVID19 outcomes among people living with HIV during the COVID19 pandemic, Chicago, IL. AIDS Behav.
2020;1-3. doi: 10.1007/s10461-020-02905-2.

29. Wilkinson L, Grimsrud A. The time is now: expedited HIV differentiated service delivery during the COVID-19 pandemic. J Int AIDS Soc. 2020;23:e25503. doi: 10.1002/jia2.25503.

30. Hussen SA, Easley KA, Smith JC, ShenviN, Harper GW, Camacho-Gonzalez $\mathrm{AF}$, et al. Social capital, depressive symptoms, and HIV viral suppresion among young black, gay, bisexual and other men who have sex with men living with HIV. AIDS Behav. 2018;22(9):3024-32. doi:10.1007/s10461-018-2105-6. 